\title{
Clinical Tip: Modified Uniportal Endoscopic Gastrocnemius Recession for Treatment of Gastrocnemius Equinus Contracture
}

\author{
Kerk Hsiang Chua ${ }^{1}$, Ewe Juan Yeap ${ }^{2}$, Keen Wai Chong ${ }^{3}$, Andrew Sands ${ }^{4}$
}

\begin{abstract}
Introduction: Gastrocnemius recession was first described by Vulpius and Stoffel in 1913 for gastrocnemius equinus contracture. Equinus contracture alters the biomechanics of the foot-ankle complex and affects other musculotendinous and ligamentous constrains in the ankle, contributing to many foot and ankle pathologies. The commonly used Strayer's technique has been modified, with the use of endoscopic approaches, to try to reduce the poor cosmesis and reduce the risk of iatrogenic injury to the sural nerve. This article aims to describe a simple uniportal technique that uses readily available arthroscopic instruments to perform endoscopic gastrocnemius recessions (EGRs).

Materials and methods: The leg is prepped and draped with a rolled towel under the lateral malleolus. A 10 mm incision is made over this point. The dissection is carried bluntly to the gastrocnemius aponeurosis. The fascia is opened and a McDonald or Freer elevator is introduced between the aponeurosis and the fascia of the soleus. A $4.0 \mathrm{~mm} \mathrm{30}$ arthroscope is inserted beneath the elevator. A half-pipe (Karl Storz, Tuttlingen) is introduced over the scope which protects the sural nerve. The length of the aponeurosis is identified from proximal to distal. At this point, the sural nerve may be visualized at the end of the pipe. A triangle knife (Smith and Nephew, Andover, MA, USA) is then introduced with the sharp edge facing the half-pipe. The knife is then flipped $180^{\circ}$ and tension is applied to the aponeurosis by dorsiflexing the foot. The aponeurosis is then released under direct arthroscopic vision.

Keywords: Arthroscopy, Gastrocnemius recession, Gastrocnemius tightness, Minimal invasive.

Journal of Foot and Ankle Surgery (Asia Pacific) (2020): 10.5005/jp-journals-10040-1117
\end{abstract}

\section{INTRODUCTION}

Gastrocnemius recession was first described by Vulpius and Stoffel in 1913 for gastrocnemius equinus contracture. ${ }^{1}$ This procedure was generally performed in the pediatric population with cerebral palsy; however, in the recent years, gastrocnemius tightness has been implicated as a contributing factor in forefoot, mid foot, and hindfoot pathologies in adults as well. ${ }^{2,3}$ It alters the biomechanics of the foot-ankle complex and affects other musculotendinous and ligamentous constrains in the ankle, causing or contributing to ulceration, plantar fasciitis, adult acquired flatfoot, hallux valgus, generalized metatarsalgia, plantar intermetatarsal neuroma, posterior tibial tendinopathy, pes planus, and Achilles tendinopathy. ${ }^{4-7}$ Di Giovanni's paper defined patients having gastrocnemius tightness when there is less than $5^{\circ}$ of ankle dorsiflexion with the knee extended. ${ }^{7}$ Meanwhile Pinney's definition included the inability to dorsiflex the ankle more than $10^{\circ}$ past neutral with the knee fully extended but which subsequently corrects with the knee flexed. ${ }^{8}$

The most commonly used method for the recession of the gastrocnemius has been that described by Strayer in 1950, which is a modification of the procedure first described in 1913. ${ }^{4}$ Subsequently, this procedure has been shown to be both safe and effective. ${ }^{9,10}$ This procedure entails incision of the gastrocnemius muscle distal to the musculoligamentous junction with sparing of the soleus muscle ensured. ${ }^{4}$ The main complications associated with this procedure include injury to the sural nerve, and poor cosmesis secondary to the large incisional wound with adhesions of the muscle to the skin. ${ }^{4,9}$ Strayer's technique has been modified, including the use of endoscopic approaches, to try and reduce the poor cosmesis and reduce the risk of iatrogenic injury to the sural nerve. ${ }^{5-7,10-14}$
${ }^{1}$ Department of Orthopaedic Surgery, KK Women's and Children's Hospital, Singapore

${ }^{2}$ Prince Court Medical Centre, Kuala Lumpur, Malaysia

${ }^{3}$ BJIOS Orthopaedics, Singapore

${ }^{4}$ Weill Cornell Medical College, New York, USA

Corresponding Author: Kerk Hsiang Chua, Department of Orthopaedic Surgery, KK Women's and Children's Hospital, Singapore, Phone: +65 62944050, e-mail: zackary.chua.k.h@singhealth.com.sg

How to cite this article: Chua KH, Yeap EJ, Chong KW, et al. Clinical Tip: Modified Uniportal Endoscopic Gastrocnemius Recession for Treatment of Gastrocnemius Equinus Contracture. J Foot Ankle Surg (Asia Pacific) 2020;7(1):28-31.

Source of support: Nil

Conflict of interest: None

Endoscopic techniques have been studied and described in the literature. Over time, in the ongoing quest for better minimally invasive surgical results, these techniques have evolved from twoportal to uniportal techniques. However, these techniques require equipment that are costly and may not readily available in all hospitals. ${ }^{5,10,11}$ The potential benefits of this approach for recession of the gastrocnemius include improved cosmesis associated with smaller incisions, reduced risk of iatrogenic injury to the sural nerve, and reduced duration of the surgery. ${ }^{5,11}$ This article aims to describe a simple uniportal technique that uses readily available arthroscopic instruments to perform endoscopic gastrocnemius recessions (EGRs). Our modification allows for successful uniportal EGRs to be done in a low-cost manner as explained further in our methodology. 


\section{Materials and Methods}

The patient is placed supine on the operating table with a thigh tourniquet applied to ensure a bloodless operating field. A sand bag is placed on the contralateral hip. The level of the portal is four fingerbreadths above the flare of the medial malleolus, roughly at the edge of the aponeurosis by palpation. An ultrasound may be used for better accuracy in finding the correct location in patients with challenging surface anatomy. ${ }^{9}$ An alternative localization way is to measure the distance from the level of the heel to the anterior ankle and this distance is extrapolated superiorly to the calcaneum. This level corresponds to the safe endoscopic zone, which was described by Carl and Barrett. ${ }^{4}$

The leg is prepped and draped with a rolled towel under the lateral malleolus. A $10 \mathrm{~mm}$ incision is made over this point. The dissection is carried bluntly to the gastrocnemius aponeurosis. The fascia is opened and a McDonald or Freer elevator is introduced between the aponeurosis and the fascia of the soleus. A $4.0 \mathrm{~mm} 30^{\circ}$ arthroscope is inserted beneath the elevator. A half-pipe (Karl Storz, Tuttlingen) is introduced over the scope which protects the sural nerve. The length of the aponeurosis is identified from proximal to distal. At this point, the sural nerve may be visualized at the end of the pipe. A triangle knife (Smith and Nephew, Andover, MA, USA) is then introduced with the sharp edge facing the half-pipe. The knife is then flipped $180^{\circ}$ and tension is applied to the aponeurosis by dorsiflexing the foot. The aponeurosis is then released under direct arthroscopic vision. A second pass may be necessary to release the median raphe. Postprocedure, the ankle should have full passive dorsiflexion to $20^{\circ}$ with the knee in full extension. The wound is closed with a single suture and the patient is allowed weightbearing as tolerated. No immobilization is required. Postop regimes can include a night splint while sleeping for 6 weeks (Fig. 1). (Please refer to Appendix for the photographs of our surgical method.)

\section{Discussion}

Endoscopic gastrocnemius resection has been widely discussed and studied by multiple investigators. ${ }^{5-9,11,12,14-17}$ These techniques show that both uniportal and two portal techniques have promising results and safety profiles.

Roukis et al. retrospectively studied prospectively collected data of 23 uniportal EGR used to treat pathologic soft tissue ankle equinus contracture in 18 patients. $^{2}$ Each patient underwent

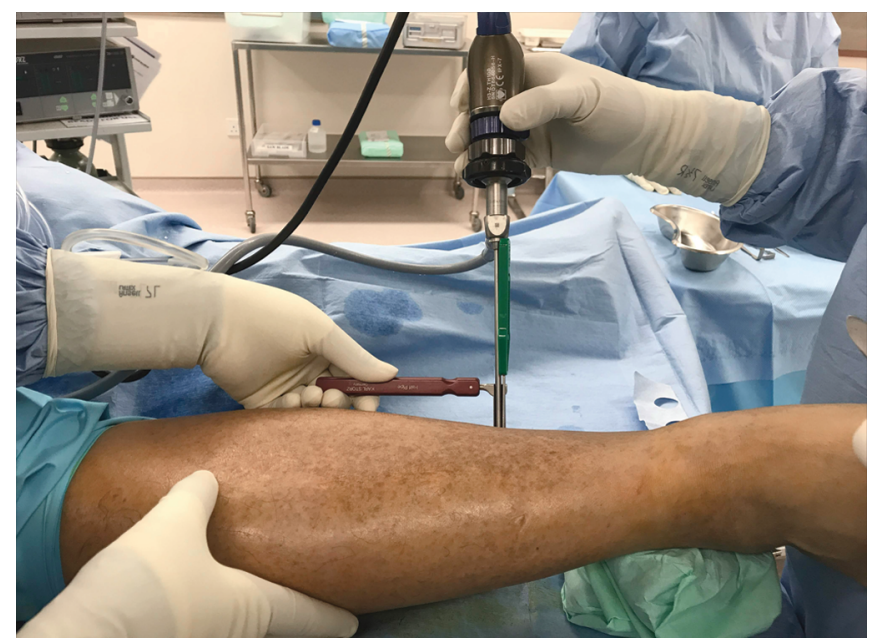

Fig. 1: Surgical technique set-up uniportal EGR using a standardized approach with an A.M. Surgical Endoscopic Release System (Wright Medical Technologies, Inc., Memphis, TN, USA). Complications were noted in nine procedures (9/23). It was also observed that three procedures showed delayed incision healing in patients with uncontrolled diabetes mellitus (3/21), three procedures with undercorrection of the soft tissue ankle equinus deformity in patients with spastic contractures $(3 / 21)$, and three procedures requiring conversion to an open gastrocnemius recession secondary to difficulty dissecting through excessive adipose tissue (3/21). The study showed that if the procedure is properly done, a uniportal technique is considered a safe and reliable minimally invasive procedure in patients with diabetes. The study advocates an open technique for patients with excessive adipose tissue and percutaneous tendo Achilles lengthening in patients with spastic contractures.

A prospective study by Thevendran et al. presented 54 patients with procedures performed on 56 feet. ${ }^{11}$ The study used a $5 \mathrm{~mm}$ portal with a scope from the Smart Release Micro Aire Endoscopic Carpal Tunnel Release System (Charlottesville, USA). In all, 11 complications (11/56) were noted, with 3 cases experiencing unsatisfactory operative scar (3/11) and 3 cases experiencing numbness over the distribution of the sural nerve (3/11). This study reported a statistically significant improvement in functional status, patient satisfaction, and pain scores. The functional scores were evaluated via the American Orthopedic Foot and Ankle Society (AOFAS) Hindfoot score, modified Olerud and Molander ( $O$ and $M$ ) score, and Short Form (SF-36). Patient satisfaction and pain scores were calculated using the modified Likert scale and visual analog scores (VAS). This study showed that this uniportal technique is both effective and safe with excellent postoperative results.

Meanwhile, Trevino et al. retrospectively studied 28 patients with 31 procedures. ${ }^{18}$ This study used a single medial or lateral portal using a 3M Agee Carpal Tunnel Release System (Micro Aire Surgical Instruments, Charlottesville, USA). Four complications (4/31) were noted. Two procedures noted that the incision for the portal was at the wrong level $(2 / 31)$, one procedure noted the failure of endoscopic approach leading to conversion to open technique to complete the gastrocnemius recession (1/31), and one procedure noted a patient developing a soft tissue infection (1/31). However, there was no incidence of sural nerve injury or Achilles tendon damage. This study reported statistically significant improvements in pain, swelling, stiffness postoperatively using the modified Olerud and Molander score $(p<0.05)$, thus proving the feasibility and safety of this uniportal technique.

Saxena et al. prospectively studied 47 patients who underwent 54 uniportal EGRs. ${ }^{17}$ Preoperatively, the extent of dorsiflexion was $-8^{\circ} \pm 4^{\circ}$. Significant improvement was observed postoperatively to $7 \pm 4^{\circ}$. There were no infections; however, six limbs noted lateral foot or leg dysesthesia (6/54), unacceptable cosmesis (6/54), one hematoma (1/54), and one overlengthened gastrocnemius (1/54). This technique can significantly improve the postoperative ankle dorsiflexion but may have potential complications.

Last but not least, Schroeder et al. prospectively studied 53 patients who underwent 60 uniportal EGR with a specifically designed uniportal endoscopic system. This technique utilized a uniportal endoscopic system with the retractable tenotomy blade (Endoscopic Gastroc Release System; Integra Life Sciences, Plainsboro, NJ, USA). Significant improvement was observed in the mean range of ankle dorsiflexion from $-2.9 \pm 1.9$ to $12.8 \pm$ 1.7. No cases of infections, hematoma, or painful scar formation 
were reported; however, in four cases (4/60) complications were observed. One case of triceps surae weakness which resolved after therapy (1/60), three cases had nervous system complications, with two experiencing transient neuritis which resolved within 5-8 weeks and one experiencing sensory loss in the distribution of the sural nerve 4 months postoperatively. This study proved the efficacy and safety profile of its purported uniportal system. ${ }^{10}$

\section{Conclusion}

This uniportal endoscopic resection technique has significant promise, as it is safe, cheap, and effective for patients with gastrocnemius equinus contracture with favorable postoperative improvements and patient satisfaction. The senior author has found this method is equivalent to the conventional methods that used to be art of his practice. Our method of EGR described can be performed with standard arthroscopic equipment. That has a significant economic advantage over other procedures, requiring costly instruments.

\section{Acknowledgment}

We would like to acknowledge Dr Akshay Kumar for his initial contribution in the literature review of this manuscript.

\section{References}

1. Vulpius O, Stoffel A. Tenotomie der end schnen der mm. Gastrocnemius el soleus mittels rutschenlassens nach Vulpius. In: Orthopadische Operationslehre. Stuttgart: Ferdinard Enke; 1913. pp. 29-31.

2. Roukis T, Schweinberger M. Complications associated with uni-portal endoscopic gastrocnemius recession in a diabetic patient population: an Observational case series. J Foot Ankle Surg 2010;49(1):68-70. DOI: 10.1053/j.jfas.2009.07.018.

3. Molund $M$, Paulsrud $\varnothing$, Ellingsen Husebye $E$, et al. Results after gastrocnemius recession in 73 patients. Foot Ankle Surg 2014;20(4):272-275. DOI: 10.1016/j.fas.2014.07.004.

4. Pinney S, Sangeorzan B, Hansen S. Surgical anatomy of the gastrocnemius recession (Strayer procedure). Foot Ankle Int 2004;25(4):247-250. DOI: 10.1177/107110070402500409.

5. Grady J, Kelly C. Endoscopic gastrocnemius recession for Treating equinus in pediatric patients. Clin Orthop Relat Res 2009;468(4): 1033-1038. DOI: 10.1007/s11999-009-1084-3.
6. Saxena A, DiGiovanniC. Ankle equinus and endoscopic gastrocnemius recession. Minimally Invasive Surgery of the Foot and Ankle. London: Springer; 2010. pp 324-325.

7. DiGiovanni CW, Kuo R, Tejwani N, et al. Isolated gastrocnemius tightness in patients without neurological impairment. J Bone Joint Surg Am 2002;84(6):962-970. DOI: 10.2106/00004623-20020600000010.

8. Pinney SJ, Hansen Jr ST, Sangeorzan BJ. The effect on ankle dorsiflexion of gastrocnemius recession. Foot Ankle Int 2002;23(1): 26-29. DOI: 10.1177/107110070202300105.

9. Yeap E, Shamsul S, Chong K, et al. Simple two-portal technique for endoscopic gastrocnemius recession: clinical tip. Foot Ankle Int 2011;32(8):830-833. DOI: 10.3113/FAI.2011.0830.

10. Schroeder S. Uniportal endoscopic gastrocnemius recession for treatment of gastrocnemius equinus with a dedicated EGR system with retractable blade. J Foot Ankle Surg 2012;51(6):714-719. DOI: 10.1053/j.jas.2012.08.002.

11. Thevendran G, Howe L, Kaliyaperumal K, et al. Endoscopic gastrocnemius recession procedure using a single portal technique: a prospective study of fifty four consecutive patients. Int Orthop 2015;39(6):1099-1107. DOI: 10.1007/s00264-015-2723-9.

12. Cychosz C, Phisitkul P, Belatti D, et al. Gastrocnemius recession for foot and ankle conditions in adults: evidence-based recommendations. Foot Ankle Surg 2015;21(2):77-85. DOI: 10.1016/j.fas.2015. 02.001 .

13. Lui T. Modified endoscopic release of gastrocnemius aponeurosis. J Foot Ankle Surg 2015;54(1):140-142. DOI: 10.1053/j.jfas.2014. 07.014.

14. Villanueva M, Iborra Á, Rodríguez G, et al. Ultrasound-guided gastrocnemius recession: a new ultra-minimally invasive surgical technique. BMC Musculoskelet Disord 2016;17(1):409. DOI: 10.1186/ s12891-016-1265-7.

15. Saxena A, Widtfeldt A. Endoscopic gastrocnemius recession: preliminary report on 18 cases. J Foot Ankle Surg 2004;43(5):302-306. DOI: 10.1053/j.jfas.2004.07.001.

16. Tashjian R, Appel A, Banerjee R, et al. Endoscopic gastrocnemius recession: evaluation in a cadaver model. Foot Ankle Int 2003;24(8):607-613. DOI: 10.1177/107110070302400807.

17. Saxena A, Gollwitzer H, Widtfeldt A, et al. Endoscopic gastrocnemius recession as therapy for gastrocnemius equinus. $Z$ Orthop Unfall 2007;145(04):499-504. DOI: 10.1055/s-2007-965385.

18. Trevino S, Gibbs M, Panchbhavi V. Evaluation of results of endoscopic gastrocnemius recession. Foot Ankle Int 2005;26(5):359-364. DOI: $10.1177 / 107110070502600503$. 


\section{Appendix}

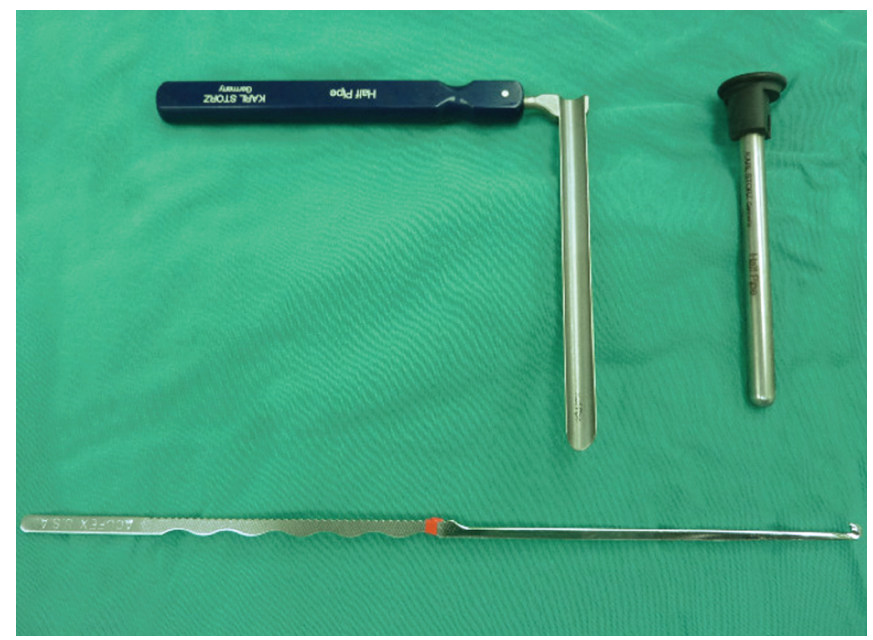

Fig. A1: Equipment required

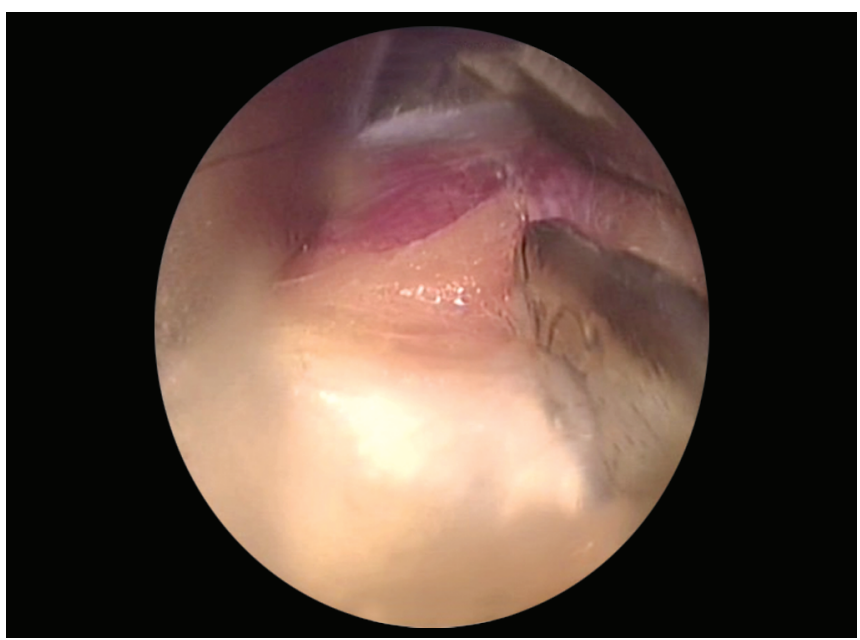

Fig. A2: Cutting

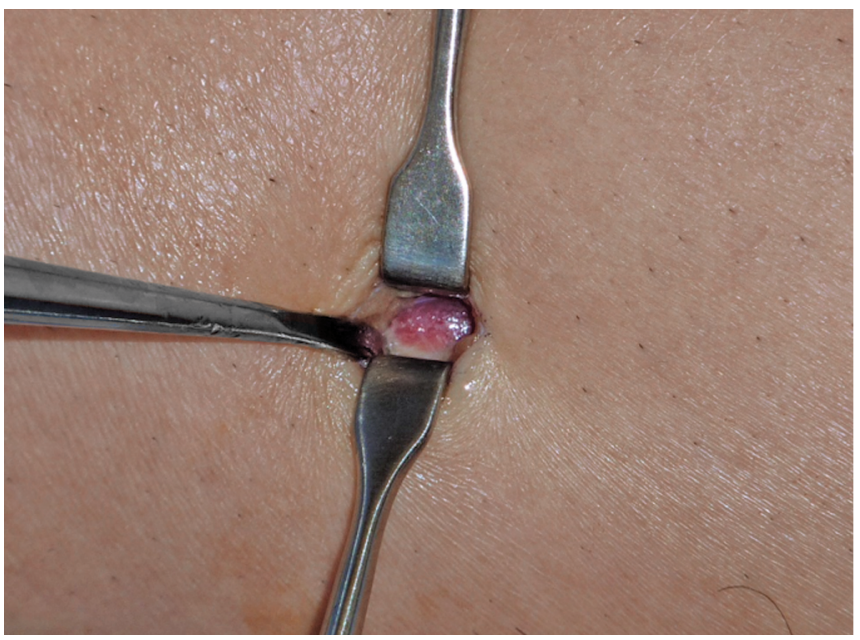

Fig. A3: Incision made

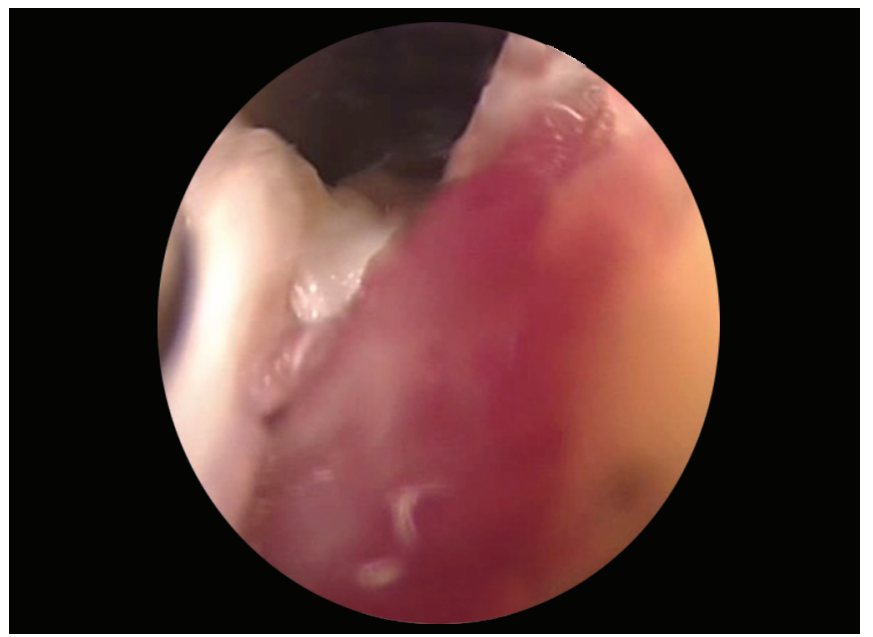

Fig. A4: Final view

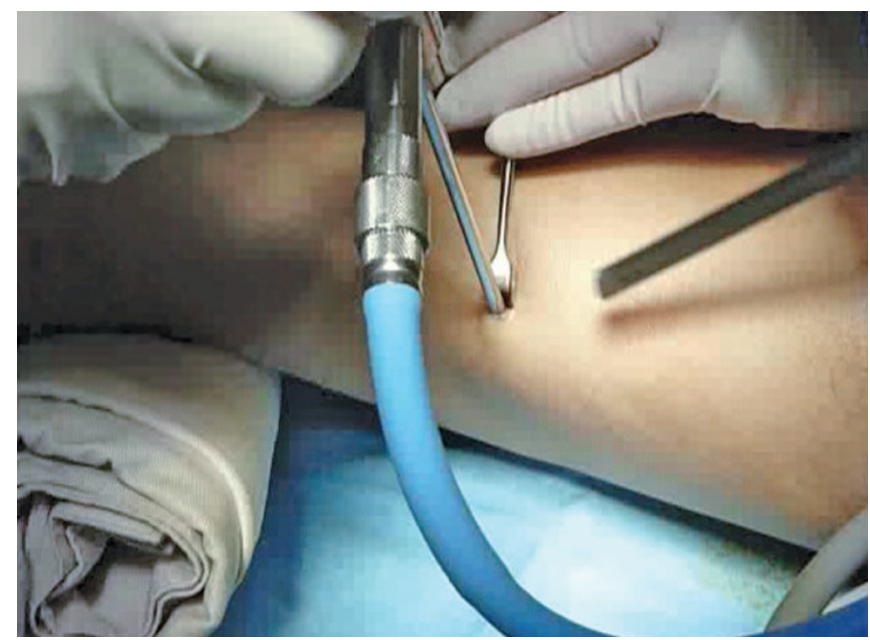

Fig. A5: Introduction of camera and sheath

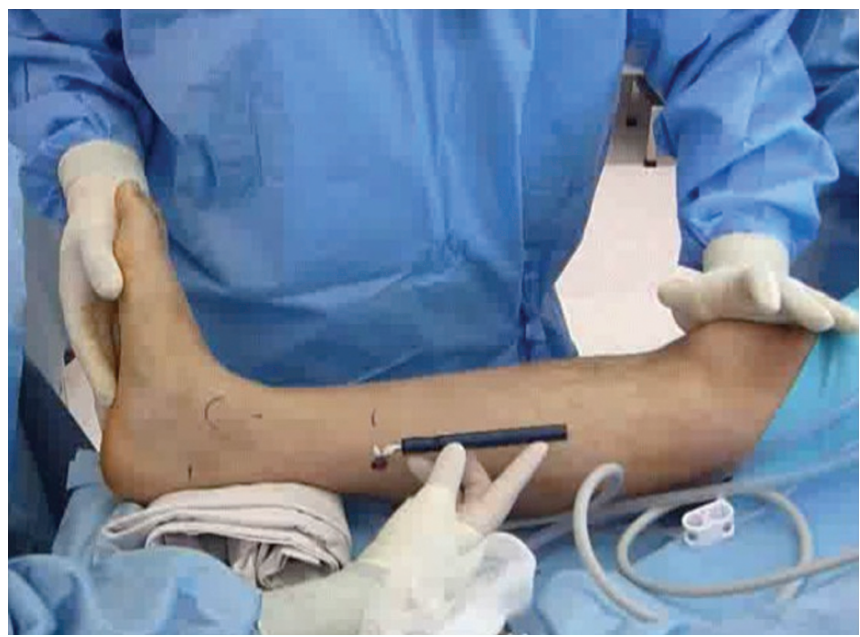

Fig. A6: Final ROM 